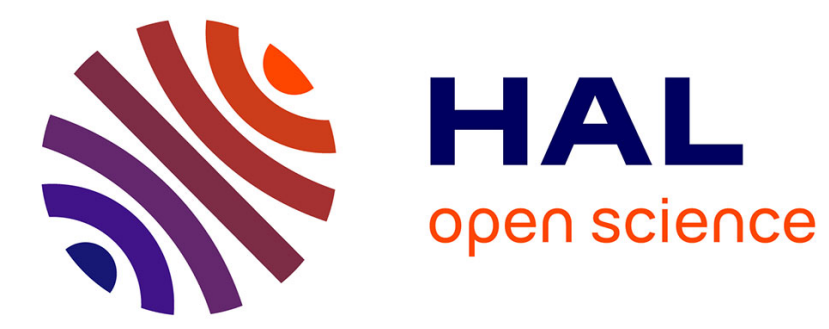

\title{
Michel Cornaton, Pierre Bourdieu. Une vie dédoublée Pascal Fugier
}

\section{- To cite this version:}

Pascal Fugier. Michel Cornaton, Pierre Bourdieu. Une vie dédoublée . 2011. hal-01672889

\section{HAL Id: hal-01672889 \\ https://hal.science/hal-01672889}

Submitted on 27 Dec 2017

HAL is a multi-disciplinary open access archive for the deposit and dissemination of scientific research documents, whether they are published or not. The documents may come from teaching and research institutions in France or abroad, or from public or private research centers.
L'archive ouverte pluridisciplinaire HAL, est destinée au dépôt et à la diffusion de documents scientifiques de niveau recherche, publiés ou non, émanant des établissements d'enseignement et de recherche français ou étrangers, des laboratoires publics ou privés. 
Pascal Fugier, «Michel Cornaton, Pierre Bourdieu. Une vie dédoublée », Lectures [En ligne], Les comptes rendus, 2011, mis en ligne le 03 mars 2011. URL : http://journals.openedition.org/lectures/1302

$1 C^{\prime}$ est une lecture personnalisée de Pierre Bourdieu qu'opère Michel Cornaton dans cet ouvrage, en portant un éclairage sur la vie du sociologue et en discutant son œuvre sociologique du point de vue, tantôt convergent, tantôt divergent, de sa propre histoire de vie et de sa propre production intellectuelle. Ainsi est-ce notamment le spécialiste de l'étude des camps de regroupement de la Guerre d'Algérie ${ }^{1}$ qui discute l'auteur, entre autre, de Sociologie de l'Algérie et du Déracinement. Mais c'est aussi, corrélativement, la trajectoire biographique d'un fils d'infirmier issu de la campagne bressane qui porte un éclairage sur celle d'un fils de receveur de La Poste au sein d'un petit village béarnais.

2Cette lecture personnalisée de Bourdieu explore donc ce qui est «en partage » (p. 83) entre les deux sociologues, l'Algérie concernant le terrain de recherche et une origine sociale populaire ainsi que l'appartenance à une même situation de génération concernant leur portrait sociologique. Mais cette lecture explore aussi, à l'inverse, tout ce qui les distingue. Or, si Michel Cornaton n'hésite pas à lancer quelques "piques" à Pierre Bourdieu, notamment quand il revient sur la détestable façon dont ce dernier l'a accueilli lors de leur première rencontre (p. 15-16), il reconnaît son « trajet universitaire exceptionnel » et tout ce qui le sépare du sien, qualifié $d^{\prime} \ll$ ordinaire » (p. 9). C'est donc une contribution originale sur Pierre Bourdieu qu'apporte l'auteur. Mais si elle évite aussi bien l'hagiographie que la diatribe, elle n'en demeure pas moins critiquable sur certains points que nous pouvons relever afin d'engager la discussion de l'ouvrage.

3Reconnaissons tout d'abord à l'auteur le mérite de ne pas se cacher derrière une posture de neutralité, supposée livrer une analyse dénuée de toute subjectivité. Ainsi, plutôt que de les refouler ou de les dénier, c'est en rendant explicites les traces singulières de son regard sociologique et de sa trajectoire qu'il part en quête de cet idéal régulateur qu'est l'objectivité. Cela dit, on peut regretter que, optant pour une telle posture autoanalytique, Michel Cornaton ne se confronte pas davantage à ce que ses recherches et sa perception de celles de Pierre Bourdieu doivent à son

\footnotetext{
${ }^{1}$ M. Cornaton, Les camps de regroupement de la guerre d'Algérie, Paris, L'Harmattan, 1998. Cet ouvrage a notamment l'intérêt de rendre visible ce fait historique que sont les camps de regroupement et qui concerne au moins 2350000 algériens, « soit $28 \%$ de la population "musulmane" » (p. 67), le nombre s'élevant à 3250000 si on prend en compte les "recasés" et les "resserrés". L'auteur indiquant malheureusement que « à ce jour, selon I'historien Gilbert Meynier, un seul manuel scolaire, français, mentionnerait leur existence » (p. 66).
} 
histoire de vie. Nous pensons par exemple à la problématique de la reconnaissance, Michel Cornaton s'efforçant de comprendre l'agressivité du jeune Bourdieu par son «mal de reconnaissance » (p. 16) sans pour autant se mettre personnellement à l'épreuve d'une telle problématique. On est pourtant tenté de jouer à "I'arroseur arrosé" face à l'insistance avec laquelle il défend la paternité de l'analyse de la persistance des camps de regroupement bien après I'indépendance de l'Algérie (p. 17-18). Idem lorsqu'il revient sur l'enracinement biographique des études kabyles menées par Pierre Bourdieu et le « sentiment de trahison » (p. 19) qu'elles entraînent vis-à-vis de ses terres natales béarnaises et qui se manifestent aussi dans un rapport malaisé avec la hauteur et la distance du point de vue scolastique. Michel Cornaton aurait pu explorer à son propre compte ces possibles tensions identitaires entre son habitus primaire et son habitus secondaire (de chercheur). Là aussi sommes-nous tentés de demander à l'auteur si et dans quelle mesure il a vécu lui-même « une vie dédoublée », pour nous référer au sous-titre de l'ouvrage. Il nous semble ainsi que $I^{\prime} \ll$ analyse comparée » (p. 9) qu'il effectue entre sa trajectoire intellectuelle et celle de Pierre Bourdieu prend avant tout la forme d'une socioanalyse de Pierre Bourdieu plutôt qu'une « auto-socianalyse » (p. 10).

4Néanmoins, au regard de la difficulté de l'exercice, nous ne pouvons que reconnaître la valeur de cette contribution socioanalytique. À I'instar du chapitre que Michel Cornaton consacre aux « guerres d'Algérie respectives » que lui et Pierre Bourdieu ont connues. Le pluriel de cet intitulé soulignant à juste titre à quel point la position que tient le chercheur sur le terrain de recherche détermine ses prises de position théoriques. Aussi, la guerre d'Algérie que voit Pierre Bourdieu, depuis «les vues d'Alger » et celles de ses « experts», n'est pas la guerre que vit Michel Cornaton «en territoire de guerre» (p. 52). De même nous semble particulièrement avisée la lecture critique que l'auteur effectue de la problématique bourdieusienne de la domination masculine (p. 134-139). Critique qui s'avère être aussi une auto-critique quand l'auteur déplore sa découverte «trop tardive » des études de la littérature berbère effectuée par Camille Lacoste-Dujardin et « de ce qu'elle dénomme la "science des femmes" qui, à travers des contes [...] s'insurge contre la domination des mythes masculins » (p. 55). Et nous pouvons aussi mentionner le lien fécond que Michel Cornaton tisse entre la trajectoire sociale dans laquelle s'inscrit Pierre Bourdieu et la conception qu'il tend à avoir des classes sociales (rejetant en l'occurrence la thèse marxiste selon laquelle il existe des classes "en soi" et "pour soi"). Michel Cornaton soutient ainsi que « ce qu'est une classe sociale Bourdieu l'a appris dans son enfance à travers l'amour pour son père, déclassé, qui n'a pas eu la chance d'appartenir à une classe laborieuse emblématique : la classe ouvrière, la classe paysanne, mais le classe bâtarde des employés » (p. 117).

5 Nous allons toutefois clôturer cette recension par une remarque critique et qui concerne le plan global de l'ouvrage. En effet, nous ne sommes aucunement convaincu de l'intérêt d'avoir placé à la fin de chaque chapitre 
les extraits d'autres auteurs, qui présenteraient « I'avantage d'approfondir une analyse ou encore de donner un point de vue décalé, en ce sens qu'il[s] apporte[nt] un éclairage différent » (p. 11). Le fait est que certains extraits, faute d'être discutés par l'auteur, apparaissent davantage "hors propos" que « décalés ». Et il aurait été préférable que Michel Cornaton mobilise cet espace afin d'expliciter davantage sa problématique et ses thèmes d'analyse, à commencer par ceux qui s'enchaînent dans son dernier chapitre, abordant en quelques pages et sans articulation la sociologie critique, l'habitus, la laïcité, les médias, le pouvoir et enfin les femmes selon Pierre Bourdieu. Rien que ça! 